\section{Antiferromagnetic order as the competing ground state in electron-doped $\mathrm{Nd}_{1.85} \mathrm{Ce}_{0.15} \mathrm{CuO}_{4}$}

\author{
H. J. Kang ${ }^{\star}$, Pengcheng Dai ${ }^{\star}$, J. W. Lynn $\neq$, M. Matsuura $\dagger$, \\ J. R. Thompson ${ }^{\star} \dagger$, Shou-Cheng Zhang $\$$, D. N. Argyriou\|, Y. Onose $\|$ \\ \& Y. Tokuraף\#行
}

* Department of Physics and Astronomy, The University of Tennessee, Knoxville, Tennessee 37996-1200, USA

$\dagger$ Condensed Matter Sciences Division, Oak Ridge National Laboratory, Oak Ridge, Tennessee 37831-6393, USA

$\ddagger$ NIST Center for Neutron Research, National Institute of Standards and Technology, Gaithersburg, Maryland 20899-8562, USA

$\$$ Department of Physics, McCullough Building, Stanford University, Stanford, California 94305-4045, USA

|| Hahn-Meitner-Institut, Glienicker Str 100, Berlin D-14109, Germany

I Spin Superstructure Project, ERATO, Japan Science and Technology, Tsukuba 305-8562, Japan

\# Correlated Electron Research Center, Tsukuba 305-8562, Japan

is Department of Applied Physics, University of Tokyo, Tokyo 13-8656, Japan

Superconductivity in the high-transition-temperature (high- $T_{\mathrm{c}}$ ) copper oxides competes with other possible ground states ${ }^{1,2}$. The physical explanation for superconductivity can be constrained by determining the nature of the closest competing ground state, and establishing if that state is universal among the high- $T_{c}$ materials. Antiferromagnetism has been theoretically predicted $^{3,4}$ to be the competing ground state. A competing ground state is revealed when superconductivity is destroyed by the application of a magnetic field, and antiferromagnetism has been observed in hole-doped materials under the influence of modest fields ${ }^{5-12}$. None of the previous experiments have revealed the quantum phase transition from the superconducting state to the antiferromagnetic state, because they failed to reach the upper critical field $B_{\mathrm{c} 2}$. Here we report the results of transport and neutron-scattering experiments on electrondoped $\mathrm{Nd}_{1.85} \mathrm{Ce}_{0.15} \mathrm{CuO}_{4}$ (refs 13,14$)$, where $B_{\mathrm{c} 2}$ can be reached ${ }^{15}$. The applied field reveals a static, commensurate, anomalously conducting long-range ordered antiferromagnetic state, in which the induced moment scales approximately linearly with the field strength until it saturates at $B_{\mathrm{c} 2}$. This and previous experiments on the hole-doped materials therefore establishes antiferromagnetic order as a competing ground state in the high- $T_{\mathrm{c}}$ copper oxide materials, irrespective of electron or hole doping.

We grew single crystals of $\mathrm{Nd}_{1.85} \mathrm{Ce}_{0.15} \mathrm{CuO}_{4}$ (NCCO) using the travelling solvent floating-zone technique ${ }^{16}$. As grown, the samples are not superconducting ${ }^{17,18}$ and exhibit the non-collinear longrange antiferromagnetic (AF) structure of undoped $\mathrm{Nd}_{2} \mathrm{CuO}_{4}$ (Fig. 1a; refs 19-21). To obtain superconductivity, the samples were annealed in an $\mathrm{Ar} / \mathrm{O}_{2}$ gas mixture ${ }^{16}$. Although antiferromagnetism in the heat-treated NCCO is greatly reduced, it could not be completely eliminated, and the residual AF order coexists with superconductivity ${ }^{22}$. Figure $1 \mathrm{c}$, d shows the temperature $(T)$ dependence of the in-plane resistivity $\left(\rho_{a b}\right)$ at zero field and the magnetic susceptibility of the sample $\left(T_{\mathrm{c}}=25 \mathrm{~K}\right.$ with a transition width of $3 \mathrm{~K})$, respectively. At $5 \mathrm{~K}, \rho_{\mathrm{c}}(B)$ measured for the $c$-axis-aligned field $\left(\mathrm{B} \|_{c}\right.$-axis) shows a field-induced superconducting-to-normal state transition at $B_{\mathrm{c} 2}=6.2 \mathrm{~T}$ (Fig. 1e). Figure 1f displays $\rho_{\mathrm{c}}(T)$ for several applied fields. In a field $(9 \mathrm{~T})$ that exceeds $B_{\mathrm{c} 2}, \rho_{\mathrm{c}}(T)$ initially decreases as temperature decreases but shows an anomalous upturn for $\mathrm{T}<12 \mathrm{~K}$, similar to $\mathrm{Pr}_{1.85} \mathrm{Ce}_{0.15} \mathrm{CuO}_{4}$ (refs 23, 24).

We use neutron diffraction to investigate the magnetic ordering, and label wave vectors $Q=\left(q_{x}, q_{y}, q_{z}\right)$ in $\AA^{-1}$ as $(H, K, L)=\left(q_{x} a / 2 \pi\right.$, $\left.q_{y} a / 2 \pi, q_{z} c / 2 \pi\right)$ in the reciprocal lattice units (r.l.u.) appropriate for the tetragonal unit cell of NCCO (space group $I 4 / \mathrm{mmm}, a=3.92 \AA$ and $c=12.07 \AA$ ). The experiments were performed in two different geometries. First, the superconducting $\mathrm{CuO}_{2}$ planes were aligned in the horizontal $(H, K, 0)$ scattering plane and the applied vertical field was along the $c$-axis $(\mathbf{B} \| c$-axis). Second, the crystal was aligned in the $[H, H, L]$ zone and the vertical field was applied along the $[1,-1,0]$ direction (B\|ab-plane). In common with other layered superconductors, a $\mathbf{B} \|_{c}$-axis field suppresses superconductivity more strongly than a $\mathbf{B} \| a b$-plane field.

Figures $1 \mathrm{~g}, \mathrm{~h}, 2 \mathrm{a}-\mathrm{f}$ and $3 \mathrm{a}-\mathrm{h}$ summarize the outcome of the

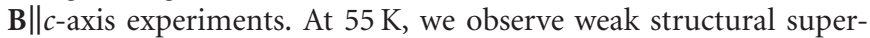
lattice peaks intrinsic to superconducting NCCO (Fig. 2a-c; ref. 25). While long-range ferromagnetic (FM) ordering is induced-as seen by the added magnetic intensity to the $(1,1,0)$ structural Bragg peak intensity-when a 6-T B $\| c$-axis field is applied (Fig. 2d), the lack of intensity change at superlattice positions (Fig. 2a-c) shows that no static AF order is induced by the field at this temperature. On cooling to $5 \mathrm{~K}$, the $\mathrm{Cu}$ spins order below a Néel temperature $T_{\mathrm{N}}$ of $\sim 38 \mathrm{~K}$ in the non-collinear structure (Figs $1 \mathrm{a}$ and $2 \mathrm{~b}$; refs 19-21). The rapid intensity increase below $15 \mathrm{~K}$ in Fig. $3 \mathrm{~b}$ at $0 \mathrm{~T}$ is due to the polarization of the $\mathrm{Nd}$ moment by strong $\mathrm{Cu}-\mathrm{Nd}$ interactions ${ }^{17-19}$. At $5 \mathrm{~K}$ and $6 \mathrm{~T}$, in addition to an enhanced FM moment at $(1,1,0)$ (Fig. 2d), field-induced signals begin to emerge at superlattice positions (Fig. 2a-c). Particularly interesting is the appearance of magnetic peaks at $(0.5,0.5,0)$ and $(0.5,0,0)$, which demonstrates that the field-induced magnetic structure differs from that in zero field (Fig. 1a), as the latter has vanishing intensity at these positions (Fig. 1g; ref. 19). A survey of reciprocal space in the $(H, K, 0)$ plane indicates clear field-induced effects that obey the selection rules $( \pm(2 m+1) / 2, \pm(2 n+1) / 2,0),( \pm(2 m+1) / 2, \pm n, 0)$, and $( \pm m, \pm(2 n+1) / 2,0)$ with $m, n=0,1,2$ (Fig. 1h). Table 1 summarizes these observations, and makes intensity comparisons with model calculations.

For AF ordered copper oxides, the magnetic scattering may have zero or non-zero intensity at $(0.5,0.5,0)$ depending on the spin arrangements along the $c$-axis, but it always vanishes at $(0.5,0,0)$. To understand the anomalous field-induced $(0.5,0,0)$ scattering, we consider four different models. First, the sample may phaseseparate into two different magnetic domains with one domain contributing to $(0.5,0.5,0)$ and the other to $(0.5,0,0)$. Second, two $\mathrm{CuO}_{2}$ sheets within the unit cell may have different magnetic structures, with one contributing to $(0.5,0.5,0)$ and the other to $(0.5,0,0)$. However, it is unclear why two identical $\mathrm{CuO}_{2}$ planes should behave differently in an external field. Third, assuming that structural superlattice reflections (Table 1) originate from lattice distortions of both $\mathrm{Cu}(\mathrm{Nd})$ and $\mathrm{O}$ (ref. 25), the field-induced effect at these positions may simply be FM ordering associated with the polarization of $\mathrm{Cu}(\mathrm{Nd})$ ions. The differences in the field-dependent data between superlattice (Fig. 3e-g) and (1, 1, 0) Bragg (Fig. 3h) reflections, though, are inconsistent with this model. Fourth, the $\mathrm{Cu}$

\begin{tabular}{|c|c|c|c|}
\hline$(H, K, L)$ & $I_{\text {lattice }}$ at $55 \mathrm{~K}$ & $I_{\text {mag }}$ at $5 \mathrm{~K}$ & $I_{\text {mag }}(\text { model } 4)^{\star}$ \\
\hline$(0.5,0.5,0)$ & 1.7 & 7.1 & 7.1 \\
\hline$(1.5,1.5,0)$ & 1.8 & 0.3 & 1.7 \\
\hline$(0,0.5,0)$ & 0.5 & 7.8 & 6.92 \\
\hline$(0,1.5,0)$ & $<0.16$ & 5.5 & 1.9 \\
\hline$(1,0.5,0)$ & 0.4 & 2.1 & 2.8 \\
\hline$(0.5,1,0)$ & 0.4 & 3.2 & 2.8 \\
\hline$(2,0.5,0)$ & 0.3 & 0.5 & 1.2 \\
\hline$(0.5,2,0)$ & $<0.16$ & 1.4 & 1.2 \\
\hline$(1,1.5,0)$ & 0.25 & 1.1 & 1.5 \\
\hline$(1.5,1,0)$ & $<0.16$ & 0.8 & 1.5 \\
\hline$(0,1,0)$ & 1.1 & $<0.16$ & 0 \\
\hline
\end{tabular}

Extinction and $\mathrm{Nd}$ absorption for the large crystal (a cylindrical rod $38 \mathrm{~mm}$ long and $3.8 \mathrm{~mm}$ in diameter) caused intensity (in arbitrary units) differences for equivalent reflections. The uncertainties for the intensities were about 0.16 .

${ }^{*}$ See text. 
moments in NCCO could be different at different sites within the $\mathrm{CuO}_{2}$ plane, partially owing to the intrinsically inhomogeneous doping by electrons. Assuming three different $\mathrm{Cu}$ moments $M_{1}, M_{2}$ and $M_{3}$ in the type-II AF structure ${ }^{19}$, we find that the spin arrangements in Fig. 1b can best describe the observed scattering (Table 1). Although this model may not uniquely describe the data, our results should stimulate future work in this direction.

a
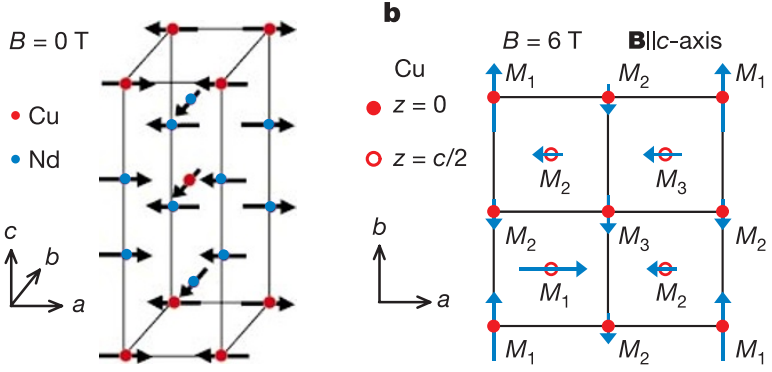

c
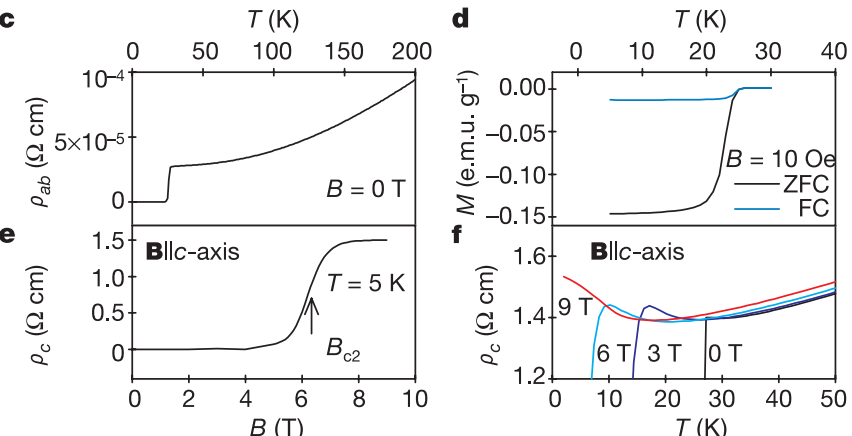

$\mathbf{9}$

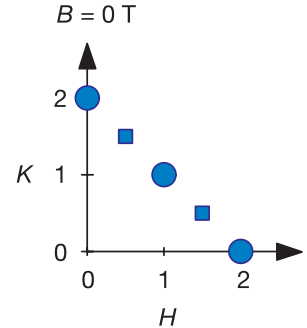

$\mathbf{h}$

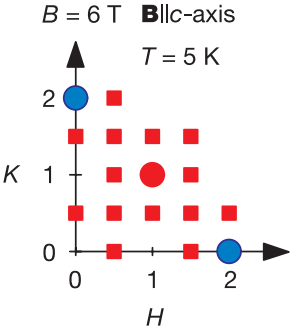

Figure 1 Spin structure models, bulk transport (magnetic) properties, and summary of reciprocal space probed in the neutron experiments. For resistivity and magnetic susceptibility, we used a 9-T physical property measurement system and a 7-T SQUID magnetometer, respectively. Our neutron-scattering experiments were performed on the BT-9 and BT-2 triple-axis spectrometers at the NIST Center for Neutron Research and on the E4 two-axis diffractometer at the Berlin Neutron Scattering Center, Hahn-MeitnerInstitute (HMI). For BT-9, the collimations were, proceeding from the reactor to the detector, $40^{\prime}-46^{\prime}-40^{\prime}-80^{\prime}$ (full-width at half-maximum), and the final neutron energy was fixed at $E_{f}=14.78 \mathrm{meV}$. Similar collimations were used for BT-2 measurements. The monochromator, analyser and filters were all pyrolytic graphite. The low- $T$ data were collected after field cooling the sample from $15-25 \mathrm{~K}$ above $T_{\mathrm{c}}$. a, The non-collinear typeI/III spin structure of the AF order in NCCO below $39 \mathrm{~K}$ (ref. 19). b, Model 4 (see text). For clarity of the AF spin arrangements, we ignored the field-induced FM moments along the $c$-axis. As we only collected data in the $(H, K, 0)$ plane for $\mathbf{B} \| c$-axis, no information was obtained for the spin arrangements along the $c$-axis direction. c, $\rho_{a b}(T)$ at 0 T. d, $T$ dependence of the zero-field-cooled (ZFC) and field-cooled (FC) magnetic susceptibility in 10-0e field. e, Magnetic field dependence of $\rho_{\mathrm{c}}$ at $5 \mathrm{~K} . \mathbf{f}, \rho_{c}(T)$ at $0,3,6$ and $9 \mathrm{~T} . \rho_{a b}(T)$ behaves similarly. The blue circles and squares in $\mathbf{g}$ show the observed fundamental nuclear and magnetic peaks, respectively, at $0 \mathrm{~T}$. In the $c$-axis spin arrangements of $\mathbf{a}$, magnetic structure factors have non-zero values only at $( \pm 0.5, \pm 1.5,0)$ and $( \pm 1.5$, $\pm 0.5,0)$ for $L=0$. For clarity, we did not plot the weak nuclear superlattice reflections. The red squares and circles in $\mathbf{h}$ illustrate the field-induced new magnetic peaks or intensity gain at $6 \mathrm{~T}$.
Figure 3 summarizes the temperature and field dependence of the neutron intensity at $(0.5,0.5,0),(0.5,1.5,0),(0.5,0,0)$ superlattice and $(1,1,0)$ structural Bragg peak positions. While the fieldinduced $\mathrm{AF}$ order peaks around $6.5 \mathrm{~T}$ at $(0.5,0.5,0)$ indicative of a phase transition from the superconducting state to an antiferromagnetically ordered state at $B_{\mathrm{c} 2}$ (Figs 2e and 3e)-the field-induced FM intensity at $(1,1,0)$ continues to rise for fields above $B_{\mathrm{c} 2}$, and does not saturate for fields up to $2 B_{\mathrm{c} 2}$ (Figs $2 \mathrm{f}, 3 \mathrm{~d}$ and $3 \mathrm{~h}$ ).

We believe that the field-induced AF signal arises from the suppression of superconductivity by the $c$-axis-aligned field. For $\mathrm{Nd}_{2} \mathrm{CuO}_{4}$, a $\mathbf{B} \| a b$-plane field induces a spin-flop transition from the non-collinear to a collinear structure ${ }^{20}$. At $0 \mathrm{~T}$ and $5 \mathrm{~K}$, magnetic scattering for the spin structure of Fig. 1a has vanishing intensity at $(0.5,0.5,0)$ and becomes very strong at $(0.5,0.5,3)$ (ref. 19). The observed $L$ dependence of the data is consistent with this spin structure (Fig. 4a-d). On applying a 7-T B $\| a b$-plane field, the zerofield intensities for $(0.5,0.5,1)$ and $(0.5,0.5,3)$ disappear. This is expected when the residual non-collinear AF order in NCCO is

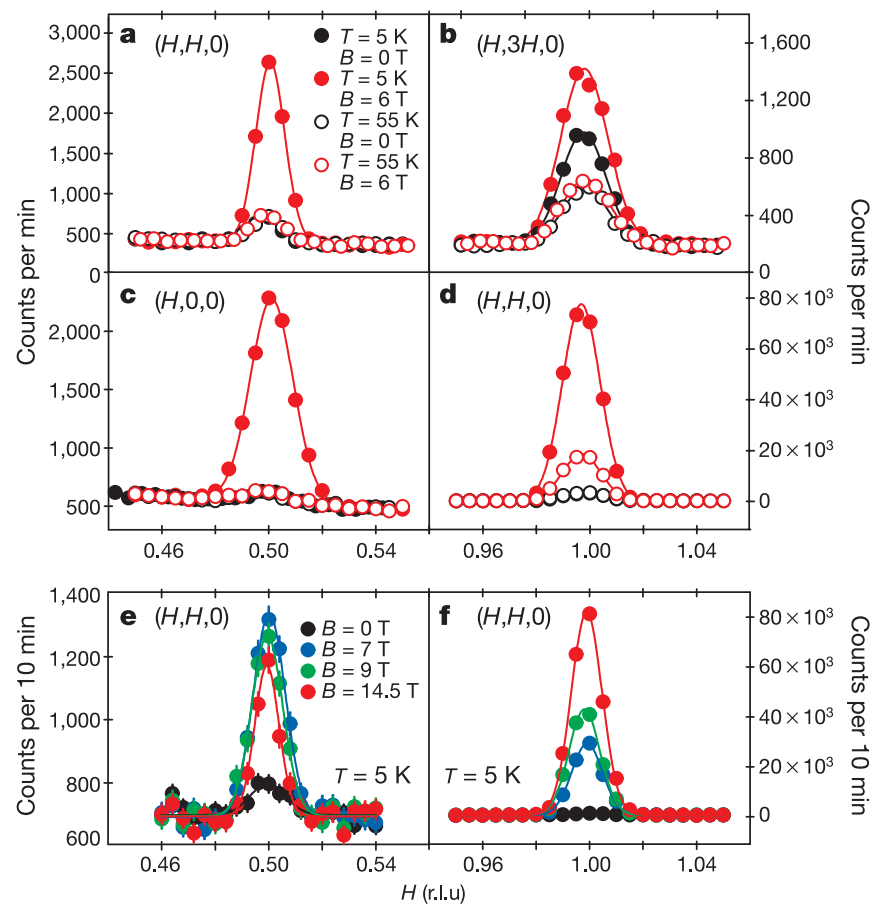

Figure 2 Effect of a $\mathbf{B} \| c$-axis field on the magnetic peaks (half integer) and induced ferromagnetic (integer) peaks below and above $T_{\mathrm{c}}$. The data in a-d were collected at NIST, whereas the high-field data in $\mathbf{e}$ and $\mathbf{f}$ were obtained on the E4 diffractometer using the VM-1, 14.5-T vertical field magnet at HMI. The collimations were $40^{\prime}-40^{\prime}$-sample$40^{\prime}$ with one pyrolytic graphite filter. Scan directions are along a, d, $(H, H, 0) ; \mathbf{b},(H, 3 H, 0)$; c, $(H, 0,0) ; \mathbf{e}, \mathbf{f},(H, H, 0)$. In a-d, the black and red filled circles represent data at $5 \mathrm{~K}$ for identical scans at zero field and 6-T field, respectively. The black and red open circles represent data at $55 \mathrm{~K}$ for identical scans at zero field and 6-T field, respectively. The Qwidths of the zero-field and field-induced scattering are resolution-limited and identical, implying an in-plane correlation length larger than $300 \AA$. The peaks in open black circles of $\mathbf{a}-\mathbf{c}$ are due entirely to structural superlattice scattering. At zero field, we confirmed the weak structural superlattice reflections reported in ref. 25 at $(H, K, L)=( \pm(2 m+1) / 2$, $\pm(2 n+1) / 2,0$ ) where $m, n=0,1$. We also observed superlattice reflections at $( \pm 0.5$, 0,0 ) type positions not allowed in the $14 / \mathrm{mmm}$ space group of NCCO. Along the $c$-axis $(L)$ direction, these superlattice peaks are very broad. At present, the microscopic origin of these superlattice reflections is unknown ${ }^{25}$.e,$(H, H, 0)$ scans through $(0.5,0.5,0)$ at $5 \mathrm{~K}$ as a function of field for fields up to 14.5T. The field-induced moment increases with increasing field up to $B_{\mathrm{c} 2}$. For fields above $B_{\mathrm{c} 2}$, the field-induced signal begins to drop due to the canting of field-induced antiferromagnetism. $\mathbf{f},(H, H, 0)$ scans through $(1,1,0)$ at $5 \mathrm{~K}$ show that the field-induced FM moment does not saturate up to $14.5 \mathrm{~T}$. 
transformed into a collinear spin structure by the applied field. For $\mathrm{Nd}_{2} \mathrm{CuO}_{4}$, the critical field for such a spin-flop transition is $0.8 \mathrm{~T}$ at $5.6 \mathrm{~K}$ (ref. 20). This is consistent with the spin-flop critical field shown in Fig. 4f. Finally, Fig. 4e shows the $T$ dependence of the scattering at $(0.5,0.5,3)$ in zero field and at $7 \mathrm{~T}$. At $7 \mathrm{~T}$, the intensity shows no $T$ dependence below $65 \mathrm{~K}$, indicating that this scattering is non-magnetic. Comparison of zero-field and 7-T data gives $T_{\mathrm{N}} \approx 38 \mathrm{~K}$ at $0 \mathrm{~T}$, a value larger than $T_{\mathrm{c}}$ but substantially smaller than that of other NCCO samples ${ }^{17,18}$. As such a field has a limited effect on superconductivity and does not change the spin moments (Fig. 4), we conclude that the field-induced AF structure seen in the
$\mathbf{B} \|_{c}$-axis geometry is a direct consequence of the suppression of superconductivity.

Understanding the quantum phase transition from the AF parent state to the superconducting state is the key issue for high- $T_{\mathrm{c}}$ copper oxides. Traditionally, this transition is achieved by chemical doping, which introduces extrinsic effects that mask the nature of the transition. Magnetic field provides an alternative, much cleaner, tuning parameter in high- $T_{\mathrm{c}}$ superconductors ${ }^{1,2}$. Although previous experiments reported enhanced AF fluctuations in the vortex state ${ }^{5-12}$, there is always a logical possibility that other forms of order are induced at higher fields, and compete predominantly with
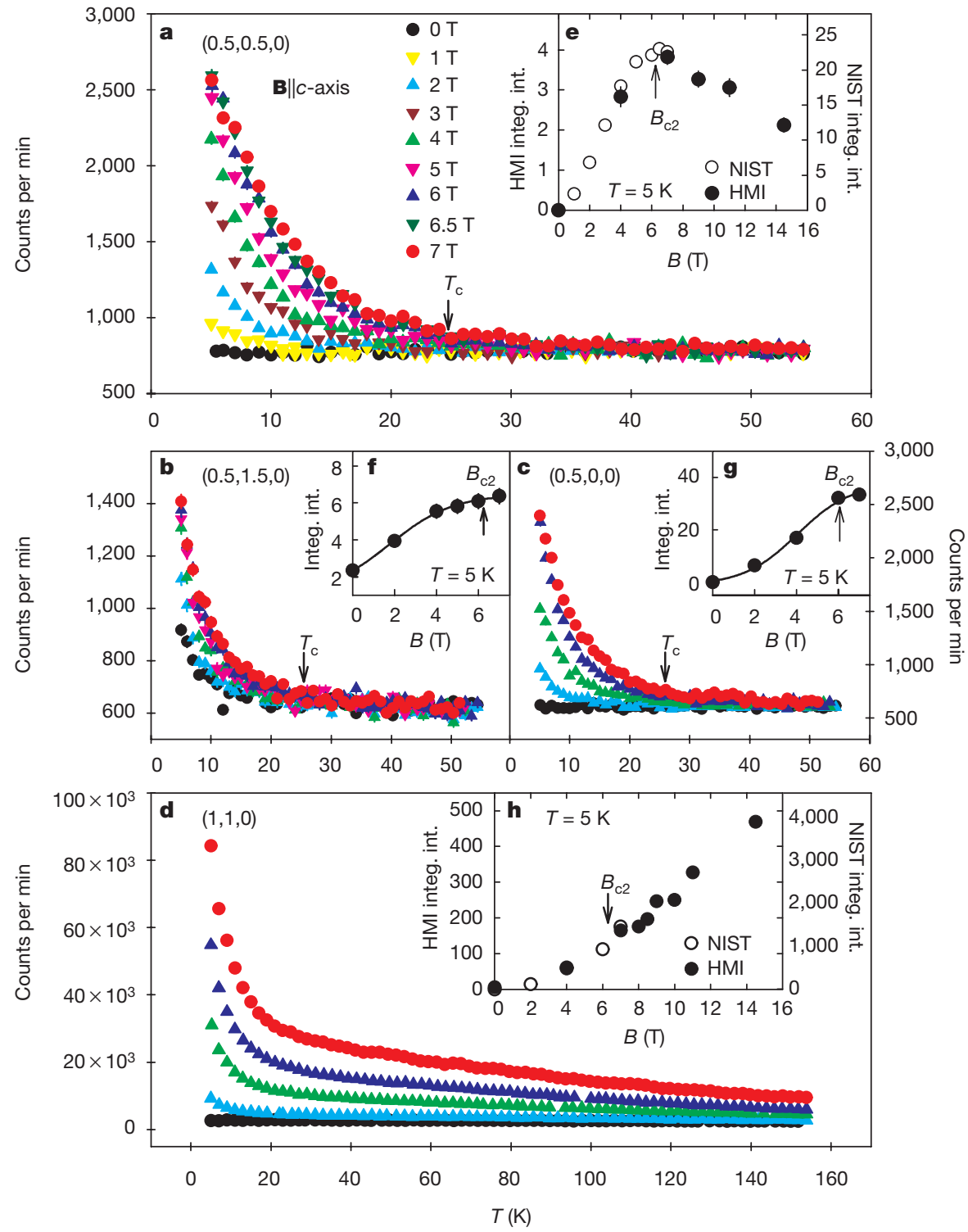

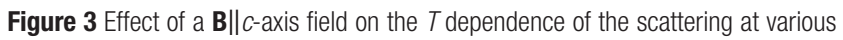
positions and the field dependence of the integrated intensity at $5 \mathrm{~K}$. a-d, $T$ dependence of the scattering at $(0.5,0.5,0),(0.5,1.5,0),(0.5,0,0)$ and $(1,1,0)$. At $0 \mathrm{~T}$, the intensity at $(0.5,0.5,0)$ in $\mathbf{a}$ is essentially temperature independent below $55 \mathrm{~K}$. On application of a field, the scattering begins to increase systematically above the zero-field value from $T \leq 8 \mathrm{~K}$ at $1 \mathrm{~T}$ to $T \leq 25 \mathrm{~K}$ at $6 \mathrm{~T}$, and finally saturates for fields around $6.5 \mathrm{~T}$. e, The field-induced integrated magnetic intensity of the $(0.5,0.5,0)$ peak as a function of applied field. The open circles are data taken at NIST whereas filled circles are results from HMI. These two sets of data are completely consistent below $7 \mathrm{~T}$. For $B>B_{\mathrm{c} 2}$, the field-induced signal decreases with increasing field due to the canting of field-induced AF moment. $\mathbf{f}, \mathbf{g}$, The field-induced scattering at $(0.5,1.5,0)$ and $(0.5,0,0)$. $\mathbf{h}$, The fieldinduced FM moment at $(1,1,0)$. The field-induced FM moment increases from $0 \mathrm{~T}$ to 14.5 T, and shows no major anomaly around $B_{\mathrm{c} 2}$. By normalizing the $T$-dependent intensity of $(0.5,0.5,0)$ and $(0.5,0,0)$ at $6 \mathrm{~T}$ with that of the $(1,1,0)$ peak at zero field, we estimate Cu moments (in $\mu_{\mathrm{B}}$ ) of $M_{1} \approx 0.19, M_{3} \approx 0.07$ and $M_{2} \approx 0.05$ at $15 \mathrm{~K}$, and Nd moments (in $\mu_{\mathrm{B}}$ ) of $M_{1 \mathrm{Nd}} \approx 0.13, M_{3 \mathrm{Nd}} \approx 0.053$ and $M_{2 \mathrm{Nd}} \approx 0.037$ at $5 \mathrm{~K}$, using the spin arrangements given in Fig. 1b. The FM field-induced moment increases with field approximately linearly up to a field close to $14.5 \mathrm{~T}$. 

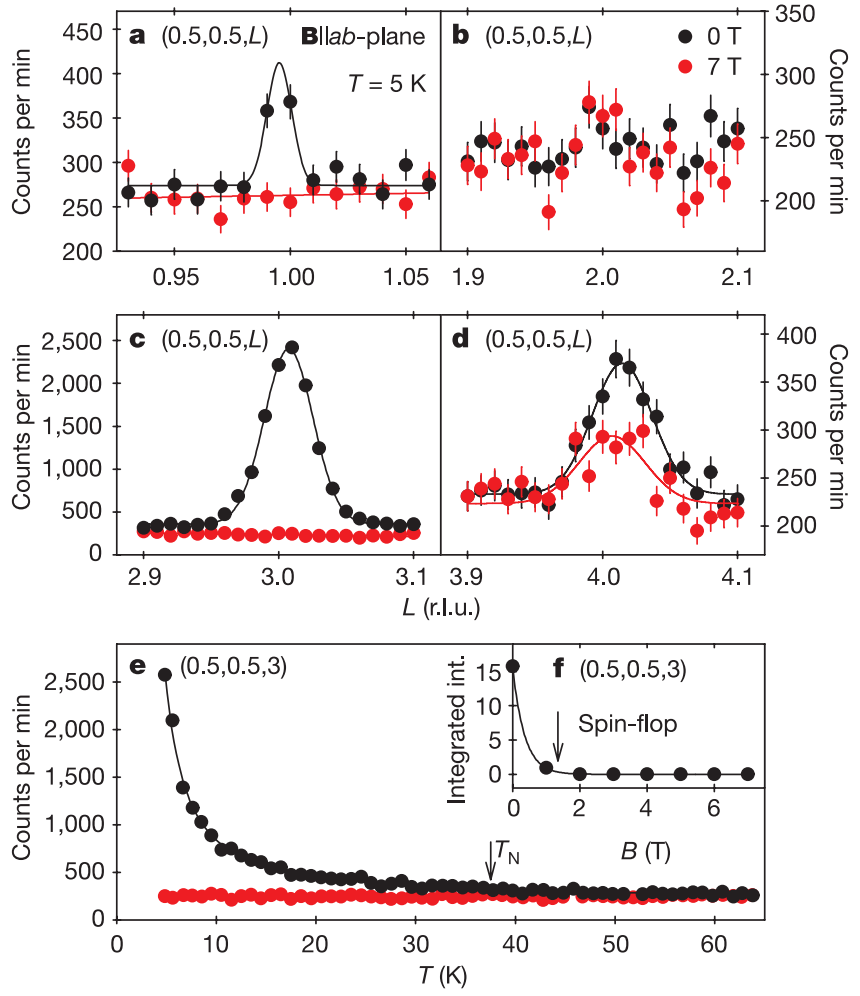

Figure 4 Effect of a $\mathbf{B} \| a b$-plane field on $(0.5,0.5, L)$ reflections. At $0 \mathrm{~T}$ and $5 \mathrm{~K}$, scans along the $[H, H, 0]$ direction show resolution-limited structural superlattice peaks around $(0.5,0.5,0)(F i g .2 b)$ and $(1.5,1.5,0)$. Along the $c$-axis $(L)$ direction, these peaks are very broad $^{25}$. a-d, Scans along the $[0.5,0.5, L]$ direction at zero (black circles) and $7 \mathrm{~T}$ (red circles). On applying a $7-\mathbf{T} \mathbf{B} \| a b$-plane field, the zero-field peak at $(0.5,0.5,3)$ disappears because of the spin-flop transition. We confirmed that a 4-T $c$-axis-aligned field enhances the $(0.5,0.5,3)$ reflection (unpublished data taken on the E4 diffractometer using the HM2 4-T horizontal field magnet at HMI). e, $T$ dependence of the scattering at $(0.5,0.5,3)$ at zero field and $7 \mathrm{~T}$ with $T_{\mathrm{N}}(\sim 38 \mathrm{~K})$ marked by the arrow. $\mathbf{f}$, The field dependence of the integrated intensity at $(0.5,0.5,3)$. The critical field for the spin-flop transition is marked by the arrow. At zero field, $M_{\mathrm{Cu}} \approx 0.016 \mu_{\mathrm{B}}$ and $M_{\mathrm{Nd}} \approx 0.01 \mu_{\mathrm{B}}$ were estimated by normalizing the integrated intensities of $(0.5,0.5,1)$ and $(0.5,0.5,3)$ to that of $(1,1,0)$ (ref. 22). At $7 \mathrm{~T}$, we obtained $M_{\mathrm{Cu}} \approx 0.012 \mu_{\mathrm{B}}$ and $M_{\mathrm{Nd}} \approx 0.01 \mu_{\mathrm{B}}$ by using $(0.5,0.5,2),(0.5,0.5,4)$ and $(1$, $1,0)$ reflections. Therefore, the spin-flop transition induced by the $7-T$ in-plane field does not change the spin moments.

superconductivity at $B_{\mathrm{c} 2}$. Our experiment demonstrates that a direct quantum phase transition from the superconducting state to an anomalously conducting antiferromagnetically ordered state is induced at $B_{\mathrm{c} 2}$. Combined with earlier reports on the hole-doped materials $s^{5-12}$, this experiment shows that AF order can be induced by an applied field irrespective of electron or hole doping. The detailed experimental results reported here, including the temperature and field dependence of the induced AF moments, provide powerful quantitative constraints on our theoretical understanding of the interplay between magnetism and superconductivity in high- $T_{\mathrm{c}}$ copper oxides.

Received 11 November 2002; accepted 1 April 2003; doi:10.1038/nature01641.

1. Levi, B. G. Magnetism and superconductivity fight for control in high- $T_{\mathrm{c}}$ superconductors. Phys. Today 55, 14-15 (2002).

2. Sachdev, S. \& Zhang, S. C. Tuning order in the cuprate superconductors by a magnetic field. Science 295, 452-454 (2002)

3. Zhang, S. C. A unified theory based on $\mathrm{SO}(5)$ symmetry of superconductivity and antiferromagnetism. Science 275, 1089-1096 (1997).

4. Arovas, D. P., Berlinsky, A. J., Kallin, C. \& Zhang, S. C. Superconducting vortex with antiferromagnetic core. Phys. Rev. Lett. 79, 2871-2874 (1997)

5. Katano, S., Sato, M., Yamada, K., Suzuki, T. \& Fukase, T. Enhancement of static antiferromagnetic correlations by magnetic field in a superconductor $\mathrm{La}_{2-} \mathrm{Sr}_{\mathrm{x}} \mathrm{CuO}_{4}$ with $\mathrm{x}=0.12$. Phys. Rev. B 62, R14677-R14680 (2000).
6. Lake, B. et al. Spins in the vortices of a high-temperature superconductor. Science 291, 1759-1762 (2001)

7. Lake, B. et al. Antiferromagnetic order induced by an applied magnetic field in a high-temperature superconductor. Nature 415, 299-302 (2002).

8. Khaykovich, B. et al. Enhancement of long-range magnetic order by magnetic field in superconducting $\mathrm{La}_{2} \mathrm{CuO}_{4+y}$. Phys. Rev. B 66, 014528 (2002).

9. Miller, R. I. et al. Evidence for static magnetism in the vortex cores of ortho-II $\mathrm{YBa}_{2} \mathrm{Cu}_{3} \mathrm{O}_{6.50}$. Phys. Rev. Lett. 88, 137002 (2002).

10. Mitrovic, V. F. et al. Spatially resolved electronic structure inside and outside the vortex cores of a high-temperature superconductor. Nature 413, 501-504 (2001).

11. Mitrovic, V. F. et. al. Antiferromagnetism in the vortex cores of $\mathrm{YBa}_{2} \mathrm{Cu}_{3} \mathrm{O}_{7-\delta}$. Preprint cond-mat/ 0202368 at $\langle$ http://xxx.lanl.gov $\rangle$ (2002).

12. Kakuyanagi, K., Kumagai, K., Matsuda, Y. \& Hasegawa, M. Antiferromagnetic ordering and disappearance of pseudogap within the vortex core of $\mathrm{Tl}_{2} \mathrm{Ba}_{2} \mathrm{CuO}_{6+\hat{\delta}}$. Preprint cond-mat/0206362 at $\langle$ http://xxx.lanl.gov $\rangle$ (2002).

13. Tokura, Y., Takagi, H. \& Uchida, S. A superconducting copper oxide compound with electrons as the charge carriers. Nature 337, 345-347 (1989).

14. Takagi, H., Uchida, S. \& Tokura, Y. Superconductivity produced by electron doping in $\mathrm{CuO}_{2}$-layered compounds. Phys. Rev. Lett. 62, 1197-1200 (1989).

15. Hidaka, Y. \& Suzuki, M. Growth and anisotropic superconducting properties of $\mathrm{Nd}_{2-\mathrm{x}} \mathrm{Ce}_{\mathrm{x}} \mathrm{CuO}_{4-\mathrm{y}}$ single crystals. Nature 338, 635-637 (1989).

16. Onose, Y., Taguchi, Y., Ishizaka, K. \& Tokura, Y. Doping dependence of pseudogap and related charge dynamics in $\mathrm{Nd}_{2-} \mathrm{Ce}_{\mathrm{x}} \mathrm{CuO}_{4}$. Phys. Rev. Lett. 87, 217001 (2001).

17. Yamada, K. et al. Neutron scattering study on electron-hole doping symmetry of high- $T_{c}$ superconductivity. J. Phys. Chem. Solids 60, 1025-1030 (1999).

18. Uefuji, T. et al. Coexistence of antiferromagnetic ordering and high- $T_{c}$ superconductivity in electrondoped superconductor $\mathrm{Nd}_{2-x} \mathrm{Ce}_{\mathrm{x}} \mathrm{CuO}_{4}$. Physica C 357, 208-211 (2001).

19. Lynn, J. W. \& Skanthakumar, S. in Handbook on the Physics and Chemistry of Rare Earths Vol. 31 (eds Gschneidner, K. A. Jr, Eyring, L. \& Maple, M. B.) 315-350 (Elsevier Science, Amsterdam, 2001).

20. Skanthakumar, S., Lynn, J. W., Peng, J. L. \& Li, Z. Y. Field-dependence of the magnetic-ordering of Cu in $\mathrm{R}_{2} \mathrm{CuO}_{4}(\mathrm{R}=\mathrm{Nd}, \mathrm{Sm})$. J. Appl. Phys. 73, 6326-6328 (1993).

21. Skanthakumar, S., Lynn, J. W., Peng, J. L. \& Li, Z. Y. Observation of noncollinear magnetic-structure for the $\mathrm{Cu}$ spins in $\mathrm{Nd}_{2} \mathrm{CuO}_{4}$-type systems. Phys. Rev. B 47, 6173-6176 (1993).

22. Uefuji, T., Kurahashi, K., Fujita, M., Matsuda, M. \& Yamada, K. Electron-doping effect on magnetic order and superconductivity in $\mathrm{Nd}_{2-x} \mathrm{Ce}_{x} \mathrm{CuO}_{4}$ single crystals. Physica C 378-381, 273-277 (2002).

23. Fournier, $\mathrm{P}$. et al. Insulator-metal crossover near optimal doping in $\mathrm{Pr}_{2-x} \mathrm{Ce}_{\mathrm{x}} \mathrm{CuO}_{4}$ : Anomalous normal-state low temperature resistivity. Phys. Rev. Lett. 81, 4720-4723 (1998).

24. Hill, R. W. et al. Breakdown of Fermi-liquid theory in a copper-oxide superconductor. Nature 414, 711-715 (2001).

25. Kurahashi, K., Matsushita, H., Fujita, M. \& Yamada, K. Heat treatment effects on the superconductivity and crystal structure of $\mathrm{Nd}_{1.85} \mathrm{Ce}_{0.15} \mathrm{CuO}_{4}$ studied using a single crystal. J. Phys. Soc. Jpn 71, 910-915 (2002).

Acknowledgements We thank R. Jin, D.-H. Lee, S.-H. Lee, H. A. Mook and S. Skanthakumar for discussions, and R. Jin and K. Prokes for experimental assistance. This work was supported by the US NSF and DOE.

Competing interests statement The authors declare that they have no competing financial interests.

Correspondence and requests for materials should be addressed to P.D. (daip@ornl.gov).

\section{Selectivity in vibrationally mediated single-molecule chemistry}

\section{J. I. Pascual ${ }^{\star} \dagger$, N. Lorente $\ddagger$, Z. Song ${ }^{\star} \$, H. Conrad ${ }^{\star}$ \& H.-P. Rust ${ }^{\star}$}

${ }^{*}$ Fritz-Haber-Institut der Max-Planck-Gesellschaft, Faradayweg 4-6, D-14194 Berlin, Germany

$\dagger$ Institut de Ciència de Materials de Barcelona-CSIC, Campus de la UAB, E-08193 Bellaterra, Spain

$\ddagger$ Laboratoire Collisions, Agrégats, Réactivité, UMR5589, Université Paul Sabatier, 118 route de Narbonne, 31062 Toulouse cédex, France

$\$$ Department of Chemistry, Brookhaven National Laboratory, Building 555, PO Box 5000, Upton, New York 11973-5000, USA

The selective excitation of molecular vibrations provides a means to directly influence the speed and outcome of chemical reactions. Such mode-selective chemistry ${ }^{1}$ has traditionally used laser pulses to prepare reactants in specific vibrational states ${ }^{2}$ to enhance reactivity ${ }^{3,4}$ or modify the distribution of product species $^{5,6}$. Inelastic tunnelling electrons may also excite molecular vibrations $s^{7,8}$ and have been used to that effect on adsorbed 